\title{
ANÁLISIS DE LA LEY DE CUADRADO INVERSO PARA MEDICIÓN PRÁCTICA DE ILUMINANCIA
}

\author{
Luis Diego Marín Naranjo
}

\begin{abstract}
Resumen
La medición práctica de la iluminancia permite verificar el nivel de iluminación en diversos tipos de ambientes utilizando fuentes artificiales de luz y además, se aplica en la calibración de patrones en fotometría. Dos conceptos fundamentales son la ley de cuadrado inverso que establece la relación inversa de la distancia entre la fuente de luz y un fotodetector, y el determinar a partir de cual distancia la fuente de luz puede considerarse puntual. Este artículo presenta parte de la teoría utilizada en un proyecto de investigación realizado en la Universidad de Costa Rica, cuyo propósito fue analizar las condiciones adecuadas de la ley de cuadrado inverso en mediciones prácticas de iluminancia. Como resultado más relevante está un análisis coherente de gran cantidad de información que estaba dispersa y no ordenada en la literatura técnica, acerca de magnitudes fotométricas relevantes como iluminancia, flujo luminoso, luminancia e intensidad luminosa, y su aplicación en mediciones. Como principales conclusiones están la verificación de la ley de cuadrado inverso considerando las dimensiones de la fuente de luz o apertura limitante, los cálculos realizados y el aporte de información ordenada para profesionales en diseño de iluminación y fotometría.
\end{abstract}

Palabras clave: fotometría, luz, iluminancia, intensidad, flujo luminoso, ley de cuadrado inverso, metrología.

\begin{abstract}
The practical measurement of illuminance allows verify the illumination level in different environments using artificial light sources and also is applied in calibration of photometric standards. Two fundamental concepts are the inverse square law which defines the inverse relationship between distance from light source to photodetector, and what the distance to consider the source like point source is. This paper resumes the theory of the final report of a research project developed at University of Costa Rica with the goal to analyze the right conditions for inverse square law in practical measurements of illuminance. The most relevant result is the coherent analysis of a big amount of information dispersed in varied technical literature, and also to present the photometric quantities like illuminance, luminous flux, illuminance and luminous intensity and their applications in measurements. The main conclusion is the verification on inverse square law considering the dimensions of light sources or limiting apertures, calculations done to support this knowledge and to supply relevant information to professionals in illumination design and photometry.
\end{abstract}

Key words: photometry, light, illuminance, intensity; luminous flux, inverse square law, metrology.

Recibido: 23 de marzo del 2009 • Aprobado: 26 de mayo del 2009.

\section{DEFINICIONES}

Se incluye aquí una serie de definiciones incluyendo el año de acuñación según (Webster, 1988).

- Ley de cuadrado inverso (1921): aseveración de que algunas magnitudes físicas, como la iluminancia, varían con la distancia desde una fuente inversamente con el cuadrado de la distancia.

- Iluminación (S XIV): acción de ser iluminado. Flujo luminoso por unidad de área sobre una superficie interceptora en un punto dado.

- Iluminancia (1938): magnitud de la iluminación realizada por la luz. Irradiancia para radiometría. 
- Intensidad (1665): magnitud de fuerza o energía por unidad de área, carga, masa o tiempo.

- Fotometría (1824): rama de la ciencia que trata con la medición de la intensidad de la luz, también la práctica de usar un fotómetro.

- Radiometría (1875): medición de la intensidad de energía radiante.

- Fuente puntual (1903): una fuente de radiación (como la luz) concentrada a un punto y considerada sin extensión espacial, y que produce ondas esféricas cuya irradiancia obedece la ley de cuadrado inverso.

- Ley de Lambert (1915): la iluminancia de una superficie que recoge rayos desde una fuente puntual es inversamente proporcional al cuadrado de la distancia entre la fuente y la superficie y proporcional al coseno del ángulo que hace el rayo con la normal a la superficie.

- Luminancia (1880): la intensidad luminosa de una superficie en una dirección dada por unidad de área proyectada.

\section{CONSIDERACIONES GENERALES DE FOTOMETRÍA}

El Sistema Interamericano de Metrología (SIM) estableció, en 1999, un plan estratégico donde uno de los aspectos era determinar las áreas para las acciones regionales y nacionales, y una de estas áreas fue la radiometría y la fotometría. Para cumplir con normas internacionales en fotometría, se deben calibrar los equipos de medición de iluminancia y el experto en fotometría debe conocer los detalles de esta magnitud para efectos de cálculos de incertidumbre y para realizar una adecuada práctica de laboratorio, además de entender las definiciones para obtener la ley de cuadrado inverso en mediciones fotométricas como la intensidad luminosa y la iluminancia.

La medición práctica de la iluminancia permite verificar el nivel de iluminación que se obtiene como resultado de un diseño de ingeniería en diversos tipos de ambientes utilizando fuentes artificiales de luz y se aplica en la calibración de patrones en fotometría. En mediciones fotométricas se obtienen valores de diversas magnitudes utilizados en el nivel de investigación científica, de control de calidad industrial y comercial, y otros.

Se utiliza un sistema de medición denominado un banco fotométrico que consiste en instrumental optomecánico como pantallas limitantes, bloqueadores de luz extraviada, lentes, espejos, una mesa óptica, sistemas detectores, así como fuentes de luz de diversa índole como fuentes incandescentes. En el Laboratorio de Fotónica y Tecnología Láser de la Escuela de Ingeniería Eléctrica de la Universidad de Costa Rica se ha desarrollado un banco fotométrico para efectos de acreditación de procedimientos, calibración, ensayo y verificación de instrumentos. A fin de mejorar el banco fotométrico se conjetura si la difracción de Fresnel desde una fuente de luz hasta el detector, produce un patrón de difracción que afecta las mediciones y cómo se relaciona con la ley de cuadrado inverso que hace que la irradiancia disminuya con el cuadrado de la distancia. Ambos conceptos de la óptica clásica son conocidos y estudiados, pero su relación directa no está bien documentada. Por eso se llevó a cabo un proyecto de investigación cuyos resultados se describen en este documento. En este se desea estudiar por qué se sugiere trabajar a distancias de más de cincuenta centímetros desde la fuente al detector, considerando la ley de cuadrado inverso para la irradiancia y si esto tiene relación con la difracción de Fresnel.

Un resultado importante es la relación entre la intensidad luminosa de una fuente de luz de área finita en dirección hacia un punto $\mathrm{P}$ y la iluminancia que esta brinda a ese punto P. Se obtiene así una relación para calcular la iluminancia suministrada por fuentes de cualquier forma y distribución de luminancia, sujeta solo a la facilidad de realizar la integración, ya sea en forma cerrada o por aproximación de computador. La intensidad es una propiedad exclusiva de una fuente puntual en una dirección dada. Si la distancia $r$ es grande, comparada con la máxima dimensión de la fuente, se puede obtener la intensidad de la fuente. Es un caso en que el fotodetector está suficientemente lejano tal, que las líneas desde 
cada elemento partiendo de la fuente hacia el punto pueden ser asumidas paralelas.

Si la distancia $r$ no es grande comparada con las máximas dimensiones de la fuente, se falla en producir la intensidad de la fuente. Se obtiene una ecuación que puede ser usada con fuentes no puntuales, pero solo si el punto detector está relativamente distante de la fuente. De aquí surge la importancia de determinar la distancia.

Se define la intensidad de una fuente de luz como el flujo dividido por el ángulo sólido. El ángulo sólido se define como el área hacia la fuente dividida por la distancia al cuadrado. a iluminancia se define de una ecuación fundamental que es la intensidad, dividida por la distancia al cuadrado.

Se deduce un importante criterio para tratar una fuente como puntual, cuando el producto de las dimensiones laterales es muy pequeño comparado con el cuadrado de la distancia a la fuente. Se concluye que se hace una aproximación de la ley de cuadrado inverso para la iluminancia, considerando que no hay una forma de medir directamente la intensidad luminosa o el flujo luminoso (excepto que se cuente con una esfera integradora o un goniómetro).

Se mide más bien la iluminancia y se utiliza la ley de cuadrado inverso para obtener estas otras magnitudes. Esto conduce a un estudio detallado de la exactitud de los cálculos de la ley de cuadrado inverso, considerando una fuente lambertiana para realizar cálculos de aproximaciones.
En los cálculos de aproximaciones de la ley de cuadrado inverso se obtienen errores que dependen de la ecuación elegida, y uno de los resultados de la investigación fue valorar la distancia óptima desde la fuente al detector para realizar una medición real en un banco fotométrico.

En el análisis de problemas ópticos se supone que la luz proviene de una fuente puntual idealizada. $\mathrm{Si}$ se desea realizar una práctica experimental donde se mida la iluminancia en condiciones geométricas conocidas a diferentes distancias, se calcula la fuente puntual virtual y se puede calcular la intensidad y la luminancia y el flujo radiante. Esta práctica experimental se realizó en un banco fotométrico y los resultados y detalles se presentan en otro artículo de esta revista.

\section{LEY DE CUADRADO INVERSO PARA LA ILUMINANCIA.}

La Figura 1 muestra la situación general de una fuente no uniforme finita radiando flujo luminoso a un punto sobre una superficie. El elemento de fuente dA se asume que tiene una luminancia $\mathrm{L}_{\mathrm{v}}$ en la dirección del punto P sobre el elemento receptor $\mathrm{dA}^{\prime}$.

La fuente emite $\mathrm{d} \Phi$ lúmenes en el ángulo sólido $\mathrm{d} \Omega$ el cual es subtendido por $\mathrm{dA}^{\prime}$ en la fuente. La línea desde la fuente al receptor hace un ángulo $\alpha$ con la normal en dA y $\beta$ con la

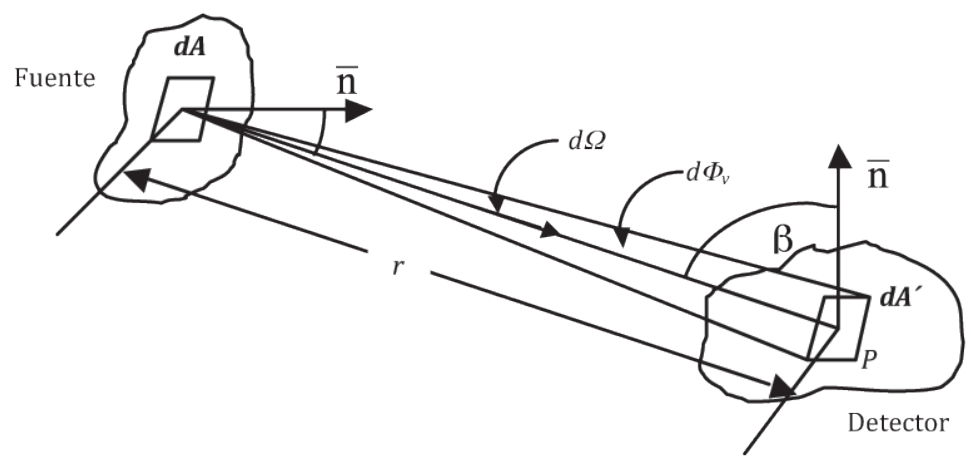

Figura 1. Situación general de la ley de cuadrado inverso. Fuente: (El autor). 
normal en $\mathrm{dA}^{\prime}$. Considerando el área proyectada se obtiene la ecuación (1).

$\mathrm{d} \Phi_{\mathrm{v}}=\mathrm{I}_{\mathrm{v}} \mathrm{d} \Omega=\frac{\mathrm{I}_{\mathrm{v}} \mathrm{dA}^{\prime} \cos \beta}{\mathrm{r}^{2}}$

La iluminancia en $\mathrm{P}$ es dad por la ecuación (2).

$\mathrm{E}_{\mathrm{v}}=\frac{\mathrm{d} \Phi_{\mathrm{v}}}{\mathrm{dA}^{\prime}}=\frac{\mathrm{I}_{\mathrm{v}} \cos \beta}{\mathrm{r}^{2}}$

Esta es la forma de la ley de cuadrado inverso para una fuente puntual. Si se considera dA como parte de una fuente de área finita, la ecuación (2) se modifica a la ecuación (3).

$d E_{v}=\frac{d I_{v} \cos \beta}{r^{2}}$

Se tiene $\mathrm{dI}_{\mathrm{v}}$ a partir de la ecuación (4).

$$
\mathrm{dI}_{\mathrm{v}}=\mathrm{L}_{\mathrm{v}} \cos \alpha \mathrm{dA}
$$

Arreglando términos se obtiene la ecuación (5).

$\mathrm{dE}_{\mathrm{v}}=\frac{\mathrm{L}_{\mathrm{v}} \cos \alpha \cos \beta \mathrm{dA}}{\mathrm{r}^{2}}$

Esta ecuación formula la ley de cuadrado inverso para un elemento en el área de una fuente. Para encontrar la intensidad de una fuente de área finita en la dirección de P y la iluminancia que brinda al punto P, se integra (4) y (5) sobre el área de la fuente para obtener las ecuaciones (6) y (7).

$$
\mathrm{I}_{\mathrm{v}}=\int_{\mathrm{S}} \mathrm{L}_{\mathrm{v}} \cos \alpha \mathrm{dA}
$$

$$
E_{v}=\int_{s} \frac{L_{v} \cos \alpha \cos \beta o s}{r^{2}}
$$

La ecuación (7) es perfectamente general y es usada para calcular la iluminancia de fuentes de cualquier forma y distribución de luminancia, sujeta solo a poder integrarla.

La ecuación (6) no es perfectamente general. La intensidad es una propiedad exclusiva de una fuente puntual en una dirección dada. Si la distancia $r$ es grande comparada con la máxima dimensión de la fuente, esta ecuación produce la intensidad de la fuente. En este caso el receptor está suficientemente lejano, tal que las líneas desde cada elemento de fuente al punto se pueden asumir paralelas.

Si la distancia $r$ no es grande comparada con las máximas dimensiones de la fuente (caso de campo cercano), la ecuación (6) falla en producir la intensidad de la fuente. Así la ecuación (6) se puede usar con fuentes no puntuales, pero solo si el detector está relativamente distante de la fuente.

\section{FORMA SIMPLE DE LA LEY DE CUADRADO INVERSO EN FOTOMETRÍA Y RADIOMETRÍA.}

Para la ecuación (6) se estableció que la intensidad luminosa se aplica solo a fuentes puntuales. El diferencial de área debe ser muy pequeño en relación con la distancia al punto donde se observa la intensidad luminosa $I_{v}$. Para incidencia perpendicular se obtiene la ecuación (8).

$$
E_{v}=\frac{I_{v}}{r^{2}}
$$

Esta es la iluminancia $E_{v}$ a una distancia $r$ desde una fuente puntual de intensidad $I_{v}$ sobre una superficie perpendicular a la línea entre la fuente puntual y la superficie donde $\mathrm{E}_{\mathrm{v}}$ se mide. Si la superficie esta inclinada, la ecuación debe 


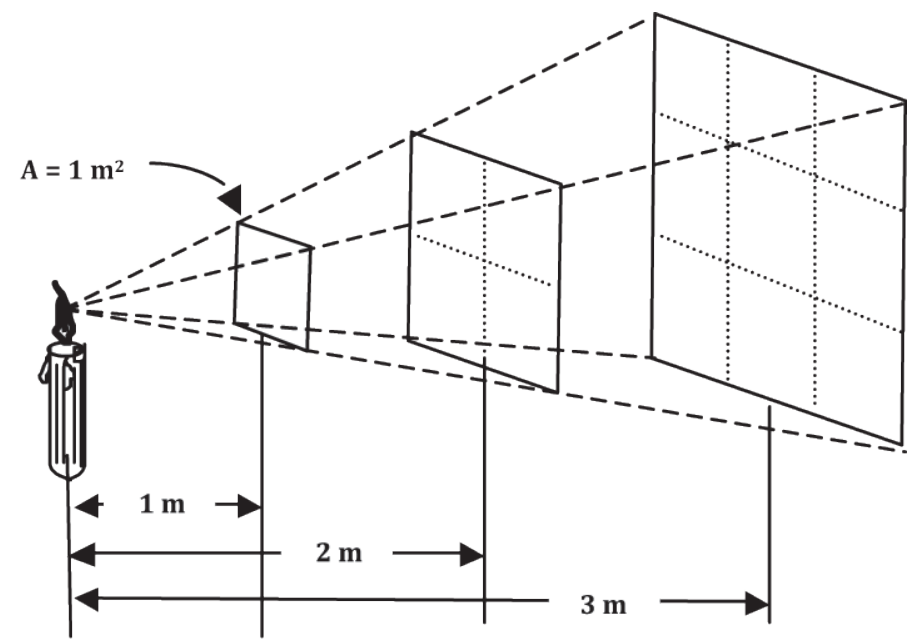

Figura 2. Geometría de la ley de cuadrado inverso para la iluminancia

Fuente (El autor).

modificarse como (2) para considerar el ángulo. Esta es la forma explícita de la ley de cuadrado inverso para la irradiancia o la iluminancia, que decrece con la distancia desde una fuente puntual, Figura 2.

La iluminancia decrece inversamente con la distancia desde la fuente. Si la luz incide sobre un área de $1 \mathrm{~m}^{2}$ a $1 \mathrm{~m}$ de la fuente, al cabo de $2 \mathrm{~m}$ cubre un área de $4 \mathrm{~m}^{2}$, a $3 \mathrm{~m}$ el área cubierta es 9 $\mathrm{m}^{2}$ y a $4 \mathrm{~m}$ se expande a $16 \mathrm{~m}^{2}$.

La ley de cuadrado inverso para la iluminancia se obtiene también considerando la propagación de radiación desde una fuente dentro de un ángulo sólido, Figura 3.

El mismo flujo pasa a través de cada área $A_{1}$, $\mathrm{A}_{2} \mathrm{y} \mathrm{A}_{3}$. Dado que estas áreas se incrementan con la distancia $r$, es evidente que hay menos flujo por unidad de área en las superficies lejos de la fuente.

Con las definiciones genéricas de intensidad $\mathrm{I}$, ángulo sólido $\Omega$ e irradiancia $\mathrm{E}$ se deriva la relación anterior entre E, I y r. El flujo se obtiene de la definición de intensidad de la fuente, ecuación (9).

$$
I=\frac{\Phi}{\Omega} \Rightarrow \Phi=I \cdot \Omega
$$

El ángulo sólido se define como:

$\Omega=\frac{\mathrm{A}}{\mathrm{r}^{2}} \Rightarrow \Phi=\frac{\mathrm{I} \cdot \mathrm{A}}{\mathrm{r}^{2}}$

La irradiancia o iluminancia a partir de (9) sobre cualquiera de las superficies se obtiene como:

$\mathrm{E}=\frac{\Phi}{\mathrm{A}}=\frac{\mathrm{I} \cdot \Omega}{\mathrm{A}}=\frac{\mathrm{I}}{\mathrm{r}^{2}}$

Es un concepto muy útil, pero deben notarse las limitaciones:

1. La fuente de la Figura 3 es una fuente puntual, que es tan pequeña que la ley de cuadrado inverso es válida. Si la fuente es extendida, la distancia $r$ desde varios puntos en la fuente a cualquier elemento de área irradiado variará, por lo que las ecuaciones simples anteriores son solo válidas para cada punto en la fuente a cada punto sobre la superficie irradiada. La irradiancia debe 
entonces ser calculada como la suma de todos los puntos en la fuente de luz.

Así, una definición más exacta de fuente puntual es: "Fuente de radiación cuyas dimensiones son suficientemente pequeñas, comparadas con la distancia entre la fuente y la superficie irradiada, para ser despreciadas en los cálculos y mediciones". Una fuente puntual que emite uniformemente en todas las direcciones se denomina fuente puntual isotrópica o fuente puntual uniforme. Una fuente ideal es la que emite isotrópicamente en el sentido que el flujo emitido es el mismo en todas las direcciones.

2. El haz de luz considerado anteriormente no está colimado, ni enfocado por lentes, ni es un haz láser, sino la propagación natural de energía radiante desde un punto en la superficie de una fuente radiando. $\underline{\mathrm{Se}}$ incorpora ahora un criterio de fuente puntual aplicado a radiometría y fotometría.

Una fuente puntual no es realmente diminuta; el mejor ejemplo son las estrellas distantes que a pesar de verse muy pequeñas, algunas tienen diámetros más grandes que la orbita de la tierra, cuando se observan desde larga distancia, como desde la tierra, pueden ser tratadas como fuentes puntuales.

El criterio para tratar una fuente como fuente puntual es que el producto de las dimensiones laterales es pequeño comparado con el cuadrado de la distancia a la fuente, Figura 4.

$d x d y \ll r^{2}$

Un criterio sugerido para decidir si una fuente finita puede ser aproximada como una fuente puntual es que la dimensión lateral máxima $d_{x}$ de una fuente sea menor que $10 \%$ de la distancia $r$ a esta. Entonces, la razón de los cuadrados de las dimensiones laterales a $\mathrm{r}^{2}$ será menor del $1 \%$ y con esto el error será del orden del $1 \%$ o menos. Esto puede servir como un criterio de aproximación cuando una fuente finita puede tratarse como una fuente puntual.

Sin embargo, si se debe cambiar $r$ para variar la irradiancia o iluminancia, una buena práctica de medición es probar el comportamiento de cuadrado inverso con las condiciones reales de medición. Para un caso de análisis, suponer un bulbo esférico con dimensiones $10 \mathrm{~cm}$ por 10 $\mathrm{cm}$. Se tiene que $10 \mathrm{~cm}=0,1 \times \mathrm{r}$ para cumplir con el criterio en una dimensión. Lo mismo en la otra dimensión.

Así $(10 \mathrm{~cm} \times 10 \mathrm{~cm})=(0,1 \times 0,1) \times \mathrm{r}^{2}$. Se despeja $r$ como $100 \mathrm{~cm}^{2} / 0,01=r^{2}$. Entonces $r=100 \mathrm{~cm}$.

En otro caso se tiene un bulbo con dimensiones $10 \mathrm{~cm}$ por $20 \mathrm{~cm}$. Así, $(10 \mathrm{~cm} \times 20 \mathrm{~cm})=(0,1 \times$ $0,1) \times r^{2}$. Se despeja $r$ como $200 \mathrm{~cm} 2 / 0,01=r^{2}$. Entonces $\mathrm{r}=141,421 \mathrm{~cm}$.

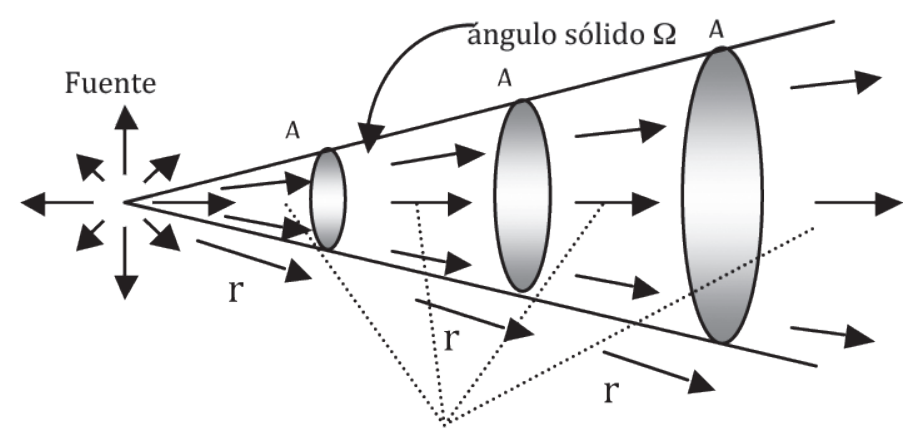

Flujo $\Phi_{\mathrm{v}}$

Figura 3. Origen de la ley de cuadrado inverso.

Fuente: (El autor). 


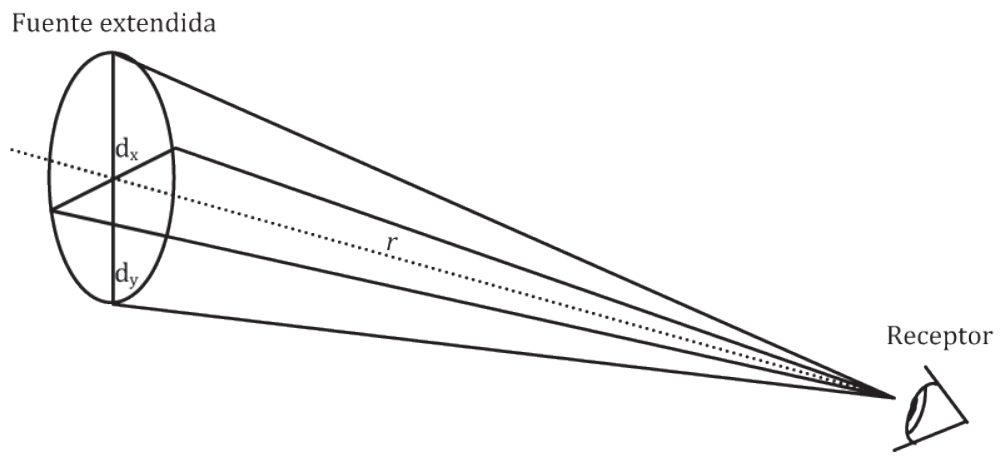

Figura 4. Geometría para definir una fuente puntual.

Fuente: (El autor).

\section{APROXIMACIONES DE LA LEY DE CUADRADO INVERSO PARA LA ILUMINANCIA}

Se mencionó que no hay una forma de medir directamente la intensidad luminosa o el flujo luminoso, a menos que se cuente con una esfera integradora o un goniómetro. Más bien se mide la iluminancia y se utiliza la ley de cuadrado inverso para obtener estas otras magnitudes. Esto conduce a un estudio adicional acerca de la exactitud de los cálculos de la ley de cuadrado inverso.

Hay que recordar que la ley de cuadrado inverso se basa en una fuente puntual. Para una fuente no puntual, se razona que la ley de cuadrado inverso puede mantenerse si la dimensión lateral máxima de la fuente es pequeña, comparada con la distancia desde la fuente al receptor.

Se estableció un criterio para la ecuación (12) de que la razón de los cuadrados de las dimensiones laterales a $\mathrm{r}^{2}$ será menor del $1 \%$. Para verificar la posible extensión de la ley de cuadrado inverso a una aplicación práctica se deben analizar diversos tipos de fuentes de luz.

En muchos casos los cálculos involucran integrales angulares y de superficie complicadas. Pero a veces la geometría es simple y una simplificación adicional se logra al asumir que las fuentes se comportan como fuentes lambertianas.

Para este efecto se considerará una fuente en forma de disco emisor proveniente de una lámpara uniforme. Se realiza el cálculo de la iluminancia
$\mathrm{E}_{\mathrm{v}}$ (irradiancia $\mathrm{E}_{\mathrm{e}}$ ) en un punto $\mathrm{P}$ a una distancia D desde el centro de la fuente. La geometría se ilustra en la Figura 5.

La fuente tiene un radio $2 \mathrm{R}$ y una luminancia constante $\mathrm{L}_{\mathrm{v}} \mathrm{cd} / \mathrm{m}^{2}$ en la dirección de $\mathrm{P}$. Se asume que $\mathrm{D}$ (distancia desde la fuente al receptor) no es muy grande comparado con $2 \mathrm{R}$, máxima dimensión lateral de la fuente. Así, la ley de cuadrado inverso no se mantiene para la fuente entera. Sin embargo, se mantiene para el anillo pequeño diferencial ilustrado en la Figura 5.

Si todos los puntos dentro del anillo, $\theta$ y la distancia desde el anillo a $\mathrm{P}$ son constantes, la forma diferencial de la ley del cuadrado inverso en la ecuación (5) se reacomoda como se presenta en la ecuación (13).

$\mathrm{dE}_{\mathrm{v}}=\frac{\mathrm{L}_{\mathrm{v}} \cos \theta \cos \theta \mathrm{dA}}{\rho^{2}}=\frac{\mathrm{L}_{\mathrm{v}} \cos ^{2} \theta \mathrm{dA}}{\rho^{2}}$

Se consideran las siguientes relaciones:

$$
\cos \theta=\frac{D}{\rho} \quad \cos ^{2} \theta=\frac{D^{2}}{\rho^{2}} \quad d A=2 \pi r d r \quad \rho^{2}=D^{2}+r^{2}
$$

Entonces:

$$
\mathrm{dE}_{\mathrm{v}}=\pi \mathrm{D}^{2} \mathrm{~L}_{\mathrm{v}} \frac{2 \mathrm{rdr}}{\left(\mathrm{D}^{2}+\mathrm{r}^{2}\right)^{2}}
$$




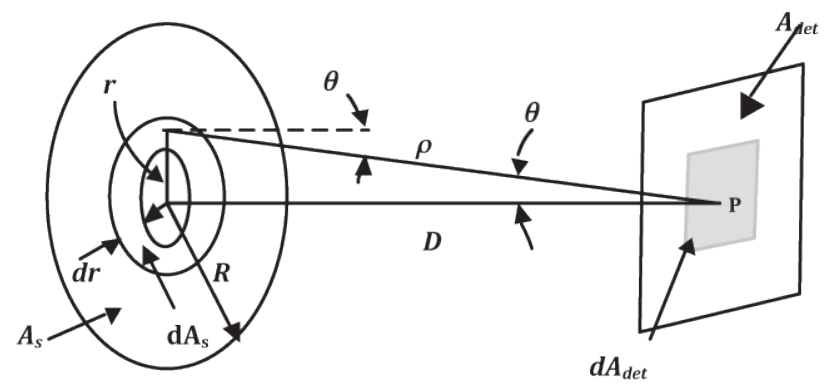

Figura 5. Geometría de una fuente luminosa en forma de disco Fuente: (El autor).

Se obtiene:

$$
E_{v}=\pi D^{2} L_{v} \int_{0}^{R} \frac{2 r d r}{\left(D^{2}+r^{2}\right)^{2}}
$$

Para integración directa se usa:

$$
\begin{aligned}
& \int \frac{x d x}{\left(c^{2}+x^{2}\right)^{n+1}}=-\frac{1}{2 n\left(c^{2}+x^{2}\right)} \\
& \left.\frac{-2}{2\left(D^{2}+r^{2}\right)}\right]_{0}^{R}=\frac{R^{2}}{D^{2}\left(D^{2}+R^{2}\right)}
\end{aligned}
$$

\section{Entonces:}

$E_{v}=\frac{\pi R^{2} L_{v}}{D^{2}+R^{2}}$

En esta última ecuación, notar que para R fijo y $\mathrm{D}$ grande, $\mathrm{E}_{\mathrm{v}}$ tiende a:

$\mathrm{E}_{\mathrm{v}} \approx \frac{\pi \mathrm{L}_{\mathrm{v}} \mathrm{R}^{2}}{\mathrm{D}^{2}}=\frac{\mathrm{I}_{\mathrm{v}}}{\mathrm{D}^{2}}$

Esto porque $I_{v}=\pi L_{v} R^{2}$ es la intensidad (flujo/ángulo sólido) de la fuente cuando está emitiendo en la dirección normal al plano, también se obtiene a larga distancia desde una pequeña fuente como (20).

$\mathrm{d} \Phi=\mathrm{I}_{\mathrm{v}} \mathrm{d} \Omega$ con $\mathrm{d} \Omega=\frac{\mathrm{dA}_{\mathrm{det}}}{\mathrm{D}^{2}}$

\section{Entonces:}

$\mathrm{d} \Phi=\frac{\mathrm{I}_{\mathrm{v}}}{\mathrm{D}^{2}} \mathrm{dA} \mathrm{det}_{\mathrm{det}}$

$\mathrm{E}_{\mathrm{v}}=\frac{\mathrm{d} \Phi}{\mathrm{dA}_{\text {det }}}=\frac{\mathrm{I}_{\mathrm{v}}}{\mathrm{D}^{2}}$

Otra forma es con la intensidad diferencial de la fuente en la dirección de P, con ecuación (4).

$$
\mathrm{dI}_{\mathrm{v}}=\frac{\mathrm{L}_{\mathrm{v}}(2 \pi \mathrm{r} \mathrm{dr}) \mathrm{D}}{\left(\mathrm{D}^{2}+\mathrm{r}^{2}\right)^{1 / 2}}
$$

Para obtener la intensidad de la fuente desde muy lejos, se escribe:

$$
I_{v}=2 \pi \pi L_{v} \int_{0}^{R} \frac{r d r D}{\left(D^{2}+r^{2}\right)^{1 / 2}}
$$


La cantidad $\mathrm{D} /\left(\mathrm{D}^{2}+\mathrm{r}^{2}\right)^{1 / 2}$ es el coseno del ángulo de la fuente. Dado que $\mathrm{I}_{\mathrm{v}}$, por definición, está en una dirección dada, $\theta$ debe ser constante, en este caso cero, durante la integración. Establecido de otra forma, D >> r para todos los valores de r. Así:

$$
I_{v}=2 \pi \pi L_{v} \int_{0}^{R} r d r
$$

Lo que es igual a:

$$
\mathrm{I}_{\mathrm{v}}=\pi \mathrm{R}^{2} \mathrm{~L}_{\mathrm{v}}
$$

Así desde muy lejos, la intensidad de la fuente es simplemente el producto de la luminancia multiplicada por el área proyectada.

\section{CONCLUSIONES}

1. La ecuación (8) define que la intensidad luminosa se aplica solo a fuentes puntuales y que el área debe ser muy pequeña en relación con la distancia al punto donde se observa la intensidad luminosa $I_{v}$. Para incidencia perpendicular se obtiene.

$$
E_{v}=\frac{I_{v}}{r^{2}}
$$

2. El criterio de las dimensiones define que $\mathrm{dx} \cdot \mathrm{dy}$ $<\mathrm{r}^{2} \mathrm{y}$ se aplica como $\mathrm{dx} \cdot \mathrm{dy}=(0,1 \times 0,1) \times \mathrm{r}^{2}$. Este criterio se aplica a cualquier tamaño de abertura que limite la luz desde una fuente que se suponga lambertiana, o sea, donde la emisión sea uniforme.

3. La aplicación al cálculo de luminancia fue de los estudios más complejos realizados. Primero el cálculo teórico de casos y luego la aplicación a varias mediciones de laboratorio utilizando un banco fotométrico simple. Un banco fotométrico típico tiene una fuente de luz estabilizada, un riel óptico de precisión, sistemas optomecánicos para desplazamiento de componentes, un sistema de detección de iluminancia calibrado y una mesa óptica para estabilidad. Las distancias de las mediciones se verifican con una cinta métrica de calidad a lo largo del riel.

4. Los resultados de las mediciones más notables son los siguientes. Se considera que se mide una fuente lambertiana y que el producto de los cuadrados de las dimensiones laterales a $r^{2}$ es menor a $1 \%$. Para obtener un error relativo bajo en la ley de cuadrado inverso, la distancia debe ser muy grande comparada con las dimensiones de la fuente de luz u obstrucción.

5. El objetivo es utilizar los valores considerados apropiados de esta distancia denominada r. Este asunto de la distancia para tener ley de cuadrado inverso se da por entendido, y se dice que debe ser más de un metro o cincuenta centímetros, pero no se justifica en la literatura especializada.

6. No es sino hasta que se realiza un estudio como este, cuando se logra un resultado convincente, aplicable a las mediciones confiables de iluminancia, su relación con la intensidad y la distancia en un banco fotométrico usando la ecuación (22), y la ecuación (26) para relacionar la intensidad con la luminancia. $\mathrm{La}$ ecuación (18) se sintetiza a la ecuación (19) considerando que las distancias son grandes comparadas con las dimensiones de la fuente de luz. Se incluyen en el informe final de la investigación (Marín, 2009), ejemplos de cálculo útiles para el curso de Licenciatura en Ingeniería Eléctrica, IE-0112 Radiometría y Fotometría.

\section{REFERENCIAS BIBLIOGRÁFICAS}

Marín, L. (2008). Informe final proyecto de investigación Determinación de la relación entre la difracción de Fresnel y la ley de cuadrado inverso en mediciones fotométricas No.322-A7-182,Vicerrectoría 
de Investigación, Universidad de Costa Rica, San José, Costa Rica.

Webster Ninth New Collegiate Dictionary. (1988). Springfield, MA: Merriam-Webster.

\section{BIBLIOGRAFÍA RECOMENDADA}

DeCusatis, C. (1998). Handbook of applied photometry. New York: Optical Society of America OSA.

Klein, M. V. \& Furtak, T. E. (1986). Optics, New York: John Wiley.

Lindsey, J. L. (1991). Applied illumination engineering. New Jersey, Prentice Hall.
McCluney, R. (1994) Introduction to radiometry and photometry. Boston: Artech House.

Murdoch, J. B. (1994). Illumination engineering. New York: Visions Communications.

\section{SOBRE EL AUTOR}

Luis Diego Marín Naranjo

Ingeniero eléctrico, M. Sc.

Coordinador del Laboratorio de Fotónica y Tecnología Láser

Escuela Ingeniería Eléctrica, Universidad de Costa Rica

San José, Costa Rica.

Teléfono: 8314-1672

Correo electrónico: 1marin@eie.ucr.ac.cr 